\section{Severe asthma and exacerbations}

\section{S60 ASPERGILLUS POLYMERASE CHAIN REACTION TESTING IN THE SEVERE ASTHMA POPULATION}

doi:10.1136/thoraxjnl-2012-202678.066

JP Farrant, H Wardman, L Holmes, L Chishimba, R Niven. University Hospital South Manchester, Manchester, England

Background Allergic Broncho-Pulmonary Aspergillosis (ABPA) is associated with known pulmonary fungal colonisation and proven response to long term anti-fungal therapy. A recent trial at our site, confirmed that anti fungal therapy was effective in a group of patients with Severe Asthma with Fungal Sensitisation (SAFS). Whether these patients have pulmonary fungal colonisation remains unknown. Previous hypotheses have indicated that bowel colonisation causes an allergic reaction leading to bronchoconstriction. Our hypothesis is that patients with SAFS, who respond to anti-fungal therapy, do have airway colonisation. Aspergillus polymerase chain reaction (APCR), represent a novel and more sensitive way to detect the presence of Aspergillus.

Methods We have performed sputum APCR tests in clinical practise over the last 2-3 years. We reviewed a retrospective cohort of patients attending a Severe Asthma Service, whose results were stored on the Mycology results system. Patient notes were reviewed to determine patient's total serum IgE's, IgE's specific to Aspergillus and anti fungal therapy status.

Results 77 results were logged on the microbiology of which 70 cases were available for notes review. Of these 44 (63\%) were female with an average age of 57.5 years. 26 had ABPA, 42 SAFS and 2 had neither ABPA nor SAFS, but had severe asthma. Overall $57 \%$ of ABPA or SAFS patients were APCR positive. $55 \%$ of SAFS patients were APCR positive, this number increased to $68 \%$ for those who were not taking anti-fungal therapy at the time of the test. The equivalent numbers for ABPA were $65 \%$ and $70 \%$ respectively. Neither of the 2 control severe patients had positive APCR. The full data is presented in table 1 .

\section{Abstract S60 Table 1}

\begin{tabular}{|c|c|c|c|}
\hline & Treatment Status & $\begin{array}{l}\text { Positive Aspergillus } \\
\text { PCR }\end{array}$ & $\begin{array}{l}\text { Negative Aspergillus } \\
\text { PCR }\end{array}$ \\
\hline \multirow[t]{2}{*}{ ABPA } & On treatment & $8(62 \%)$ & $5(38 \%)$ \\
\hline & Off treatment & $7(70 \%)$ & $3(30 \%)$ \\
\hline \multirow[t]{2}{*}{ SAFS } & On treatment & $10(43 \%)$ & $13(57 \%)$ \\
\hline & Off treatment & $15(68 \%)$ & $7(32 \%)$ \\
\hline Control & Off treatment & $0(0 \%)$ & $2(100 \%)$ \\
\hline
\end{tabular}

Conclusion This data supports the concept that patients with Severe Asthma with Fungal Sensitisation (SAFS) have pulmonary colonisation with Aspergillus. Suppression of such colonisation and reduced allergic response may then be the mechanism of action for anti-fungal therapy.

\section{S61 CLINICAL OUTCOMES AND INFLAMIMATORY BIOMARKERS IN CURRENT SMOKERS AND EX-SMOKERS WITH SEVERE ASTHMA}

doi:10.1136/thoraxjnl-2012-202678.067

${ }^{1} \mathrm{NC}$ Thomson, ${ }^{1} \mathrm{R}$ Chaudhuri, ${ }^{2} \mathrm{LG}$ Heaney, ${ }^{3} \mathrm{C}$ Bucknall, ${ }^{4} \mathrm{RM}$ Niven, ${ }^{5} \mathrm{CE}$ Brightling, ${ }^{6} \mathrm{AN}$ Menzies-Gow, ${ }^{7} \mathrm{AH}$ Mansur, ${ }^{1} \mathrm{C}$ McSharry. ${ }^{1}$ Respiratory Medicine, Institute of Infection, Immunity and Inflammation, University of Glasgow, Glasgow, UK; ${ }^{2}$ Centre for Infection and Immunity, Queen's University of Belfast, Belfast, UK; ${ }^{3}$ Glasgow Royal Infirmary, Glasgow, UK3; ${ }^{4}$ University of Manchester University Hospital of South Manchester, Manchester, UK; ${ }^{5}$ Institute for Lung Health, University of Leicester, Leicester, UK;
${ }^{6}$ Royal Brompton Hospital, London, UK; ${ }^{7}$ Birmingham Heartlands Hospital, University of Birmingham, Birmingham, UK

Rationale Some clinical outcomes are worse in current smokers and ex-smokers with mild to moderate asthma compared to never smokers, but little is known about the influence of smoking status in severe asthma.

Objectives The objectives of the analysis were to examine the association of current or previous cigarette smoking with clinical and inflammatory variables in severe asthma.

Methods We compared patient demographics, disease characteristics and biomarkers of inflammation in never smokers $(n=461$, $62.3 \%)$, ex-smokers $(n=210,28.4 \%)$ and current smokers $(n=69$, $9.3 \%)$ with refractory asthma $(n=760)$ recruited to the British Thoracic Society Severe Asthma Registry.

Results Current smokers had poorer asthma control, more unscheduled health care visits, more rescue courses of oral steroids and higher anxiety and depression scale scores than ex-smokers or never smokers. Compared to never smokers, current smokers had a reduced proportion of sputum eosinophils $(3.75 \%$ and $1.25 \%$ respectively) and lower fraction of exhaled nitric oxide $\left(\mathrm{Fe}_{\mathrm{NO}}\right)$ values (35 ppb versus $14 \mathrm{ppb}$ ), whereas ex-smokers had an increased proportion of sputum neutrophils ( $43.8 \%$ versus $56.9 \%$ ), but similar proportion of sputum eosinophils $(2.8 \%)$ and $\mathrm{Fe}_{\mathrm{NO} 50}$ values (35 ppb). Both current and ex-smokers had reduced serum specific IgE levels to some common environmental allergens.

Conclusion Current smokers with severe asthma exhibit worse outcomes for a range of clinical and health care variables compared to exsmokers and never smokers with severe asthma. Inflammatory profiles in sputum and blood differ between current smokers, ex-smokers and never smokers, possibly constituting separate phenotypes of severe asthma, which may influence responses to targeted therapies.

\section{S62 A SYSTEMATIC REVIEW OF FACTORS ASSOCIATED WITH FUTURE ASTHMA ATTACKS TO INFORM A RISK ASSESSMENT QUESTIONNAIRE}

doi:10.1136/thoraxjnl-2012-202678.068

'JD Blakey, ${ }^{2} \mathrm{~K}$ Woulnough, ${ }^{2} \mathrm{AC}$ James, ${ }^{2} \mathrm{~J}$ Fellows, ${ }^{3} \mathrm{M}$ Obeidat, 'V Navaratnam, ${ }^{1} \mathrm{~T}$ Stringfellow, 'ZW Yeoh, ${ }^{4}$ Pavord, ${ }^{5} \mathrm{M}$ Thomas, ${ }^{6} \mathrm{~S}$ Walker. 'University of Nottingham, Nottingham, UK; ${ }^{2}$ SevereBrittle Asthma Unit, Heartlands Hospital, Birmingham, UK; ${ }^{3}$ University of British Columbia, Vancouver, Canada; ${ }^{4}$ University of Leicester, Leicester, UK; ${ }^{5}$ University of Southampton, Southampton, UK; ${ }^{6}$ Asthma UK, London, UK

Asthma attacks occur across all subtypes and severities of disease. Accurate quantification of asthma attack risk would enable targeting of interventions with a potential reduction in morbidity, mortality, and costs. We aimed to develop a risk assessment questionnaire underpinned by a systematic literature review.

We searched major databases up to February 2012 using the terms: asthm* AND (exacerbati* OR admission) AND (risk OR predic* OR associat*) NOT review.

Included studies were those in individuals $>12$ years old with doctor-diagnosed asthma on treatment. Outcomes were asthma attacks defined as: deterioration of symptoms, fall in objective measures of airflow, and need for a short course of augmented asthma therapy OR admission to hospital OR attendance at emergency department. Statistical analysis was carried out using RevMan 5 and SPSS 19.

3536 unique publications were retrieved. After title screening and comparison of abstracts against a standard checklist and age criteria, data extraction was undertaken in duplicate on the remaining 143 papers (see figure).

Of 18 major research themes identified, 8 factors had a consistent and clinically important $(\mathrm{OR}>1.25)$ association with asthma attacks across publications free from major bias. The questionnaire derived from these factors is shown below. Simplified weighting is proportional to the effect size when expressed as an odds ratio (in brackets). 